\title{
Verbessert eine Heparinbeschichtung die Biokompatibilität von Poly(D,L)Laktid?
}

\section{Journal Article}

Author(s):

Heidemann, W.; Gerlach, K.L.; Fischer, J.H.; Jeschkeit, S.; Ruffieux, K.; Wagner, M; Jung, H.; Wintermantel, E.; Krüger, G.

Publication date:

1998

Permanent link:

https://doi.org/10.3929/ethz-b-000422541

Rights / license:

In Copyright - Non-Commercial Use Permitted

Originally published in:

Biomedizinische Technik 43(s1), https://doi.org/10.1515/bmte.1998.43.s1.434 


\title{
Verbessert eine Heparinbeschichtung die Biokompatibilität von Poly(D,L)Laktid?
}

\author{
Heidemann W. ${ }^{1}$, Gerlach K.L. ${ }^{1}$, Fischer J.H. ${ }^{2}$, Jeschkeit S. ${ }^{2}$ Ruffieux K. ${ }^{3}$, Wagner M. ${ }^{4}$, Jung \\ H. ${ }^{4}$, Wintermantel E. ${ }^{3}$, Krüger, G.
}

\author{
'Universitätsklinik für Mund-, Kiefer- und Gesichtschinurgie Hs.19, Leipziger Str.44, 39120 Magdeburg(Deutschland) \\ ${ }^{2}$ Institut für Experimentelle Medizin, Universität zu Köln (Deutschland) \\ ${ }^{3}$ Professur für Biokompatible Werkstoffe und Bauweisen der ETH Zürich (Schweiz) \\ 'Institut für Pathologie, Universität zu Köln (Deutschland)
}

\section{EINLEITUNG}

Polylaktide werden seit mehr als 2 Jahrzehnten allein oder in Verbindung mit anderen Polymeren, insbesondere Polyglykoliden, zur Entwicklung resorbierbarer Osteosynthesematerialien in Tierversuchsreihen und klinischen Studien getestet $[1,2,3,4,5,6]$. Bisher wurde aber noch kein Material gefunden, das die Anforderungen der initial ausreichenden Stabilität bis zur Belastbarkeit im Frakturbereich und der anschließenden Resorption ohne Fremdkörperreaktionen erfüllt [7].

Die vollständige Resorption von Implantaten aus amorphem, spritzgegossenem Poly(D,L)Laktid (PDLLA) nach 56 Wochen mit Beibehaltung von $80 \%$ der Ausgangsfestigkeit über 8 Wochen konnte in einer in-vivo Studie nachgewiesen werden [8]. Auffallig war aber die bindegewebige Einscheidung der Implantate, die eine verzögerte Resorption und damit möglicherweise entzündliche Spätreaktionen verursachen könnte. In dieser Studie wurde untersucht, ob eine Beschichtung der Oberfläche des PDLLA mit Heparin zu einer Verminderung der Kapselbildung um die Implantate und somit zu einer Verbesserung der Biokompatibilität beitragen kann.

\section{MATERIAL UND METHODEN}

50 spritzgegossene Stäbchen $(20 \times 3 \times 2 \mathrm{~mm})$ und 50 Würfel $(2 \times 3 \times 3 \mathrm{~mm})$ aus reinem Poly(D,L)lactid (PDLLA, R207) mit einem mittleren Molekulargewicht von $95.000 \mathrm{~g} / \mathrm{mol}$ wurden nach der von Seifert et. al.[9] angegebenen Methode bei Raumtemperatur zwei Stunden in einer 1:1 Mischung (pH 5,2) aus 12 prozentiger Heparinlösung und 6 prozentiger Glutaraldehydlösung aufbewahrt. Pro Implantat wurden 40mg Heparin verbraucht, die Anlagenung des Heparins an der Oberfläche wurde mit einer ToluidinBlaufärbung nachgewiesen. Die Sterilisation der Proben erfolgte durch $\gamma$-Bestrahlung mit einer Dosis von 20kGY. In Äther $/ \mathrm{O}_{2}$-Narkose wurden unter sterilen Bedingungen je ein Stäbchen und ein Würfel aus heparinbeschichtetem PDLLA und als Kontrollgruppe je ein Stäbchen und ein Würfel aus spritzgegossenem PDLLA ohne Beschichtung in Längsrichtung in die paraspinale Rückenmuskulatur von 50 männlichen Wistar-Ratten implantiert.
$2,4,6,8,12,16,20,24,28,32,36,40,44$ und 52 Wochen nach Implantation wurden von je 3 nach Zufallskriterien ausgewählten Ratten die Stäbchen für werkstoffkundliche Tests und die Würfel mit dem umgebenden Gewebe für histologische Untersuchungen entnommen. Bei der Auswertung der histologischen Schnitte wurde halbquantitativ ( $0=$ keine; $1=$ geringe; $2=$ mäßige; $3=$ deutliche; $4=$ starke zelluläre Reaktion) die Zahl der Makrophagen, Fremdkörperriesenzellen und Lymphozyten und die Ausprägung der periimplantären Bindegewebsbildung ermittelt.

\section{ERGEBNISSE}

Alle Implantate heilten reizlos ein; lediglich bei einem Tier mit multiplen entzündlichen Gelenkverändenungen traten Abszesse an den Implantationsorten der PDLLAund heparinbeschichteten PDLLA-Proben auf. Die Länge der PDLLA-Stäbchen blieb unabhängig von der Oberflächenbeschichtung bis zur 12. Woche konstant und nahm dann kontinuierlich ab; die Degradation verlief von der 20.bis zur 32. Woche bei den heparinbeschichteten Proben schneller, in der Folgezeit langsamer als der Abbau der unbeschichteten PDLLAProben(Abb.1).

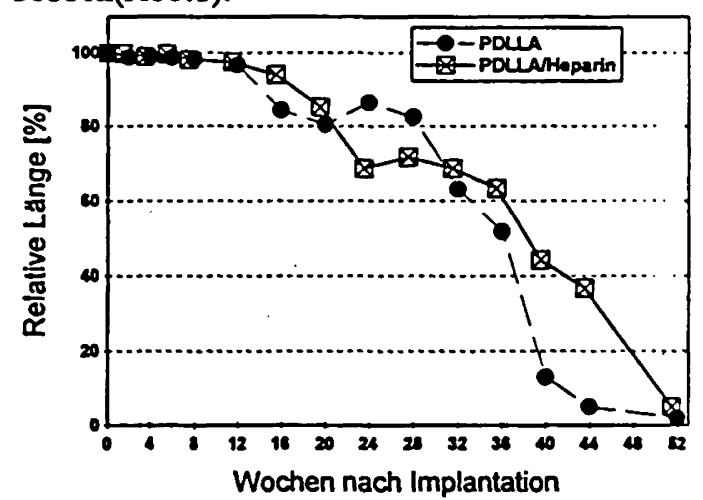

Abb.1 Längenabnahme der implantierten Stäbchen (Mittelwert der Messungen von jeweils 3 Proben)

Die Messungen des Implantatgewichts bestätigen diese Beobachtung, auch hier waren bis zur 12. Woche nach Implantation keine Veränderungen feststellbar; danach stieg das Gewicht der unbeschichteten PDLLA-Proben durch Wasseraufnahme bis zur 20 . Woche auf $158,1 \%$ des Ausgangsgewichts an und nahm anschließend bis zum Ende der Beobachtungszeit ab. Bei den 
heparinbeschichteten Implantaten war durch eine geringere Wasseraufnahme eine langsame Gewichtszunahme bis auf $123,6 \%$ des Ausgangsgewichts in der 28. Woche meßbar, anschließend nahm das Gewicht kontinuierlich ab (Abb. 2).

Die Biegefestigkeit betrug nach 8 Wochen noch etwa $80 \%$ der Ausgangsfestigkeit und fiel danach schnell ab.

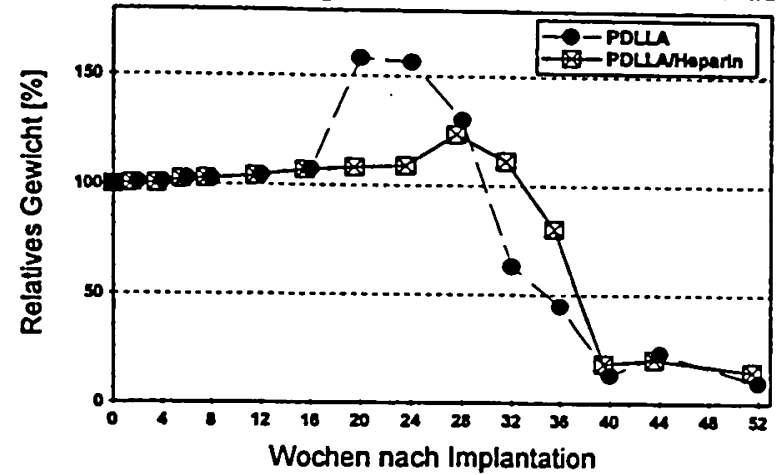

Abb.2 Änderung des Implantatgewichts (Mittelwert der Messungen von jeweils 3 Proben)

Ein signifikanter Unterschied in der periimplantären Bindegewebsbildung bestand zwischen den unbeschichteten und heparinbeschichteten Implantaten nicht; insgesamt war eine geringe bis mittlere Bindegewebsbildung in beiden Gruppen erkennbar (Abb.3).

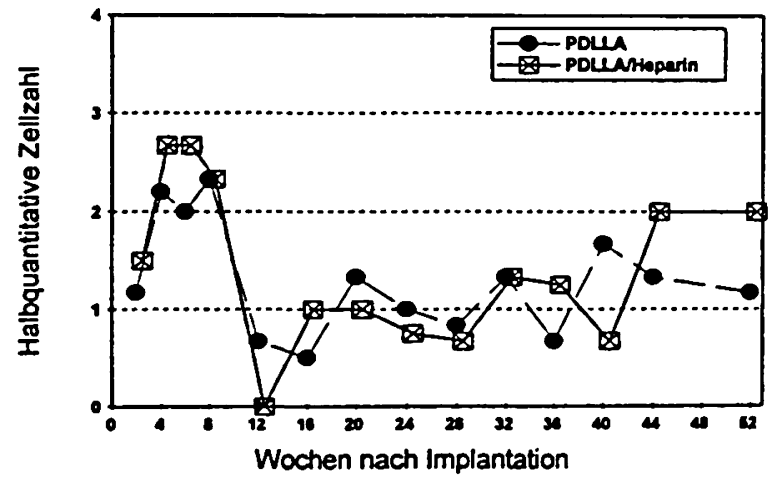

Abb.3 Bindegewebsbildung in der Umgebung der Implantate

Makroskopisch waren keine Entzündungsreaktionen in der Umgebung der unbeschichteten und beschichteten Implantaten sichtbar. Bei den histologischen Untersuchungen lag die Zahl der Makrophagen in beiden Gruppen zwischen den Graden 1 und 4 (geringe bis starke zelluläre Reaktion), die Zahl der Fremdkörperriesenzellen und Lymphozyten zwischen den Graden 0 und 3 (keine bis deutliche zelluläre Reaktion). Die Zahl der Lymphozyten war zwischen der 16. und 40. Woche nach Implantation in der Umgebung der heparinbeschichteten PDLLA-Proben im Vergleich zu den unbeschichteten PDLLA-Proben verringert (Abb.4).

\section{DISKUSSION}

Die Implantate heilten komplikationslos ein und wurden innerhalb eines Jahres bis auf kleine, nicht kristalline Reste vollständig abgebaut. Der Abbau der heparinbeschichteten Implantate verlief im Endstadium der Degradation langsamer als der Abbau der unbeschichteten PDLLA-Proben, wie die Messungen der Längen- und Gewichtsänderung der Stäbchen zeigten.

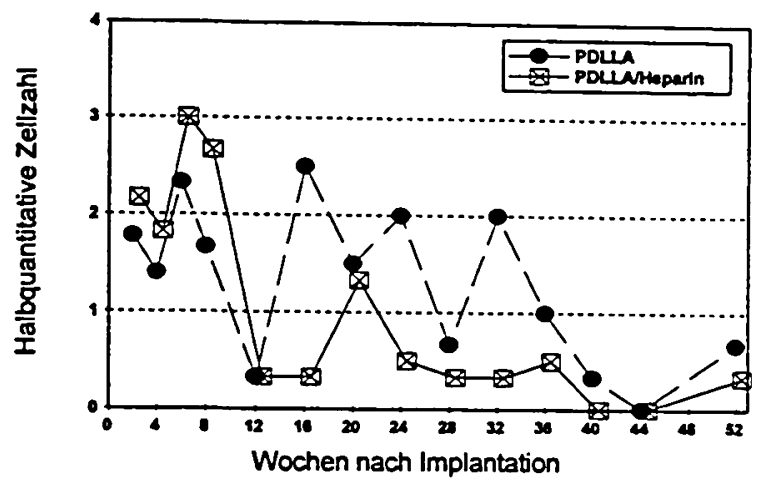

Abb.4 Halbquantitative Lymphozytenzahl im periimplantären Gewebe

Die entzündliche Gewebereaktion unterschied sich in beiden Gruppen nicht signifikant, bei den heparinbeschichteten Proben fiel eine geringere Lymphozytenansammlung zwischen der 16. und 40. Woche im periimplantären Gewebe auf. Eine Verringerung der bindegewebigen Einscheidung der PDLLA-Proben durch die Heparinbeschichtung war nicht erkennbar

Während Seifert et al. (1995) eine Verbesserung der Hämokompatibilität von PDLLA-Katheterspitzen durch eine Heparinbeschichtung sahen, konnte in dieser Studie ein günstiger Einfluß der Heparinbeschichtung auf die Histokompatibilität von PDLLA-Implantaten nicht nachgewiesen werden.

\section{LITERATUR}

[1] Getter, L.; Cutright, D.E.; Bhaskar, S.N.; Augsburg, J.K. J Oral Surgery $30: 344-348$ (1972)

[2] Bos, R.R.M.: Poly(L-lactide) Osteosynthesis development of bioresorbable bone plates and screws. Thesis, Groningen 1989

[3] Suuronen, R; Pohjonen, T.; Vasenius, J.; Vainionpää, $S$.

J Oral Maxillofac Surg 50: 255-262 (1992)

[4] Gerlach, K.L.; Clinical Materials 13: 21-8 (1993)

[5] Bessho, K.; Ilizuka, T.; Murakami, K.

J Oral Maxillofac Surg 55(9): 941 -5 (1997)

[6] Eppley, B.L.; Sadove, A.M.; Havlik, R.J.: Plast Reconstr Surg 100: 1-7 (1997)

[7] Bergsma, J.E.; de Bruijn, W.C.; Rozema, F.R.; Bos, R.R.M.; Boering, G.; Biomaterials 16: 25-31 (1995)

[8] Heidemann, W.; Gerlach, K.L.; Fischer, J.H.; Ruffieux, K.; Wintermantel, E.; Jeschkeit, S.: Biomedizinische Technik Band 41, Ergänzungsband 1: $408-9$ (1996)

[9] Seifert, B.; Groth, TH.; Herrmann, K.; Romaniuk, P.:J Biomater Sci Polymer Edn Vol.7 No.3 277.287 (1995) 